\title{
Las Marcgraviaceae de Colombia: inventario, diversidad, endemismo y distribución
}

\section{The Colombian Marcgraviaceae: checklist, diversity, endemism and distribution}

\author{
Diego Giraldo-Cañas
}

\section{RESUMEN}

Se presenta un análisis y el catálogo de las Marcgraviaceae con el objetivo de contribuir al conocimiento de la flora colombiana. El autor realizó numerosas expediciones de campo a las nueve grandes regiones naturales de Colombia y además, consultó colecciones de los herbarios CAUP, CEPEC, COAH, COL, CR, CUVC, CHOCO, F, HFAB, HUA, HUQ, IBGE, JAUM, MEDEL, MEXU, MO, MOL, MPU, NY, PSO, RSA, SI, SP, UDBC, UIS, US, VALLE y VEN. Las Marcgraviaceae están representadas en Colombia por 55 especies y ocho géneros. El endemismo corresponde a once especies. Los géneros más ricos son Marcgravia (19 especies), Souroubea (10), Marcgraviastrum (9) y Schwartzia (7). Durante el desarrollo de esta investigación, se descubrieron diez nuevas especies y un nuevo género (Pseudosarcopera) para la ciencia. La región andina, el Chocó biogeográfico y la Amazonia son las más ricas en especies, con 41, 21 y 16 especies, respectivamente.

Palabras clave: Flora de Colombia; Flora neotropical; Inventarios de flora; Marcgraviaceae.

\begin{abstract}
An analysis and the checklist of Colombian Marcgraviaceae are given, in order to contribute to knowledge of Colombian flora. This research is based on personal inventories in the nine greatest natural regions of Colombia and on the study of collections of different herbaria (CAUP, CEPEC, COAH, COL, CR, CUVC, CHOCO, F, HFAB, HUA, HUQ, IBGE, JAUM, MEDEL, MEXU, MO, MOL, MPU, NY, PSO, RSA, SI, SP, UDBC, UIS, US, VALLE, and VEN). The Marcgraviaceae are represented in Colombia by 55 species and eight genera. There are eleven species endemic to Colombia. The largest genera are Marcgravia (19 species), Souroubea (10), Marcgraviastrum (9), and Schwartzia (7). As a partial results of this research, ten new species and one genus (Pseudosarcopera) were described. The more diverse Colombian regions are Andes, Biogeographic Chocó and Amazonia, with 41, 21, and 16 species, respectively.
\end{abstract}

Keywords: Colombian flora; Marcgraviaceae; Neotropical flora;

Plant inventories.

\section{INTRODUCCIÓN}

Las Marcgraviaceae son una familia poco conocida, tradicionalmente ubicadas en el orden Parietales (Font Quer 1964) o en el orden Theales (Cronquist 1981, 1988), aunque
* Instituto de Ciencias Naturales, Facultad de Ciencias, Universidad Nacional de Colombia, Bogotá, Colombia. e-mail:dagiraldoc@unal.edu.co Recibido: 18 de febrero de 2011 Aceptado: 5 de abril de 2011 
La familia Marcgraviaceae es de distribución neotropical y consta de cerca de 134 especies, distribuidas en ocho géneros (Giraldo-Cañas 2007). Su distribución más septentrional la constituyen los estados de Chiapas, Oaxaca y Veracruz en México, mientras que la más austral corresponde a los estados de Paraná, Rio Grande do Sul y Santa Catarina en Brasil (Giraldo-Cañas 1999, Giraldo-Cañas y Pica 1999).

Las Marcgraviaceae son lianas epífitas o terrestres, o arbustos epífitos y hemiepífitos, y generalmente están restringidas a los bosques húmedos a pluviales de las regiones amazónica, andina, guayanesa, así como en el Chocó biogeográfico y Mesoamérica (sobre todo Costa Rica y Panamá), con un mayor endemismo en el noroeste de Sudamérica (la Amazonia occidental, los Andes septentrionales y el Chocó biogeográfico) (Giraldo-Cañas 1999, Picca y GiraldoCañas 1999). Por otra parte, los factores ambientales que más influyen en la diversidad y la distribución de las Marcgraviaceae son la humedad y la altitud (Giraldo-Cañas 1999).

Esta familia se caracteriza por presentar dos tipos de nectarios, que corresponden a nectarios extra-reproductivos laminales (glándulas presentes en la superficie abaxial de las hojas $=$ «hypophyllous glands») y nectarios reproductivos (brácteas nectaríferas presentes en los pedicelos, en la base de estos, en el raquis o en la base de la flor). Estos últimos corresponden a los nectarios nupciales extraflorales o recipientes nectaríferos ascidiiformes, y son los que más llaman la atención por su gran tamaño, posición, color y variedad de formas; además, son de gran importancia taxonómica en las Marcgraviaceae. El presente aporte pretende contribuir al estudio de la rica flora neotropical y en especial, al conocimiento de la familia Marcgraviaceae y la flora de Colombia.

\section{MATERIALES Y MÉTODOS}

Expediciones de campo y muestreos. Se realizaron 96 viajes a sendas localidades en las nueve grandes regiones naturales de Colombia (Amazonia, Andes, Caribe, Chocó biogeográfico, Guayana, Orinoquia, Sierra Nevada de Santa Marta, valle del río Cauca y valle del río Magdalena), entre los años 1995 y 2011, en donde se inventariaron especies de la familia Marcgraviaceae, en áreas naturales (conservadas y/o alteradas en diferentes etapas sucesionales) y en áreas rurales. Los muestreos se realizaron en forma aleatoria y cualitativa, y comprendían la recolección de ejemplares con base en los estándares de inventarios florísticos y de preservación de las muestras (Johnston 1941, Lawrence 1962).

A pesar de haber realizado tantas salidas de campo, en muy pocas ocasiones se pudo detectar la presencia de representantes de esta bella familia neotropical, lo que hace pensar en su escasez y en el bajo número de individuos por población. Los ejemplares recolectados se determinaron taxonómicamente en el Herbario Nacional Colombiano (COL), en donde se encuentran depositados bajo la serie de colección de D. Giraldo-Cañas. El inventario se complementó con el estudio de colecciones de los herbarios CAUP, CEPEC, COAH, COL, CR, CUVC, CHOCO, F, HFAB, HUA, HUQ, IBGE, JAUM, MEDEL, MEXU, MO, MOL, MPU, NY, PSO, RSA, SI, SP, UDBC, UIS, US, VALLE y VEN [abreviados de acuerdo con Holmgren et al. (1990)], y adicionalmente, se consultaron numerosas fuentes bibliográficas.

Concepto de especie. Se adoptó el concepto morfológico de especie, con base en los postulados de Crisci (1994), McDade (1995), Wiens y Servedio (2000), Uribe Meléndez (2008) y Giraldo-Cañas (2010).

Modelo del catálogo. La información del catálogo (Anexo 1) está discriminada en nueve componentes, así: 1 . Género; 2. Número de especies del género; 3 . Especie; 4 . Sinónimos; 5. Referencia (Ref.) que respalda la presencia de la especie en Colombia (en la mayoría de los casos está referida a un espécimen testigo y el herbario en donde éste se encuentra depositado o en su defecto, a una obra publicada en la que se cita la especie para Colombia); 6. Región o regiones en las que crece la especie (Amazonia, Andes, Caribe, Chocó biogeográfico, Guayana, Orinoquia, Sierra Nevada de Santa Marta, valle del río Cauca y valle del río Magdalena); 7 Gradiente altitudinal en el que crece la especie (gradientes discriminados con una amplitud de $500 \mathrm{~m}$ cada uno); 8 . Departamentos (Dept.) en los que se ha reportado la especie (AMA: Amazonas, ANT: Antioquia, ARA: Arauca, ATL: Atlántico, BOL: Bolívar, BOY: Boyacá, CAL: Caldas, CAQ: Caquetá, CAS: Casanare, CAU: Cauca, CES: Cesar, CHO: Chocó, COR: Córdoba, CUN: Cundinamarca, GUA: Guaviare, GUI: Guainía, GUJ:La Guajira, HUI:Huila, MAG:Magdalena, MET: Meta, NAR: Nariño, NSA: Norte de Santander, PUT: Putumayo, QUI: Quindío, RIS: Risaralda, SAP: San Andrés, Providencia y Santa Catalina, SAN: Santander, SUC: Sucre, TOL: Tolima, VAL: Valle del Cauca, VAU: Vaupés, VIC: Vichada); 9. Estatus de la especie (cosmopolita, endémica, introducida-cultivada, introducida-naturalizada, nativa, nativa-cultivada); 10 . Nombres vulgares; 11 . Nombres indígenas; 12. Usos; 13. Notas. En unos pocos casos se recopiló información de usos y nombres populares en Colombia, en castellano y en algunas lenguas indígenas colombianas; no obstante, esta información es muy escasa y, quizás, refleja el poco conocimiento y la baja apropiación de las Marcgraviaceae en la vida cotidiana de los pobladores de las diferentes regiones naturales en Colombia.

En relación con la consideración de los intervalos altitudinales, hay que destacar que estos se discriminaron arbitrariamente con una amplitud de $500 \mathrm{~m}$, aunque esta elección se hizo con base en el uso frecuente de intervalos con esta amplitud en diferentes investigaciones en gradientes altitudinales (Stevens 1992, Lægaard 1999, Etter y van Wyngaarden 2000, Grytnes y Vetaas 2002, Kessler 2002, 


\section{Bioetnia Volumen 8 No 1 (enero-junio), 2011}

Tabla 1

Los géneros de Marcgraviaceae en el neotrópico y en Colombia y su número de especies

\begin{tabular}{lrrc}
\hline \multirow{2}{*}{ Género } & \multicolumn{3}{c}{ Número de especies en } \\
\cline { 2 - 4 } & neotrópico Colombia & $\begin{array}{c}\text { endémicas } \\
\text { en Colombia }\end{array}$ \\
\hline Marcgravia L. & Ca. 60 & 19 & 2 \\
Souroubea Aubl. & 20 & 10 & 1 \\
Marcgraviastrum & & & \\
(Wittm. ex Szyszyl.) & & & \\
de Roon \& S. Dressler & 15 & 9 & 2 \\
Schwartzia Vell. & 19 & 7 & 5 \\
Sarcopera Bedell & 8 & 5 & 0 \\
Ruyschia Jacq. & 9 & 3 & 1 \\
Norantea Aubl. & 1 & 1 & 0 \\
Pseudosarcopera & & & \\
Gir.-Cañas & 2 & 1 & 0 \\
Total & Ca. 134 & 55 & 11 \\
\hline
\end{tabular}

Tabla 2

Diversidad de Marcgraviaceae en las regiones naturales de Colombia

\section{Región natural}

Número de especies*

\begin{tabular}{lr}
\hline Andes & 41 \\
Chocó biogeográfico & 21 \\
Amazonia & 16 \\
Guayana & 11 \\
Orinoquia & 5 \\
Caribe & 4 \\
Valle del río Magdalena & 4 \\
Sierra Nevada de Santa Marta & 3 \\
Valle del río Cauca & 3 \\
Total & 55 \\
\hline
\end{tabular}

* La suma de la columna derecha es superior a 55 especies, porque muchas especies están presentes en varias regiones naturales.

Oommen y Shanker 2005, Suárez Mayorga y Lynch 2008, Giraldo-Cañas 2010, Jørgensen et al. 2011, Larsen et al. 2011a, 2011b), lo que facilita las comparaciones de riqueza, diversidad y endemismo entre diferentes grupos biológicos. El siguiente es el modelo adoptado:

1. Pseudosarcopera Gir.-Cañas

2. Una especie
3. Pseudosarcopera diaz-piedrahitae (Gir.-Cañas) Gir.Cañas

4. Sin.: Schwartzia colombiana de Roon \& Bedell, Schwartzia diaz-piedrahitae Gir.-Cañas

5. Ref.: M. Monsalve 484 (COL, JAUM, MO, NY).

6. Chocó biogeográfico

7. $0-500 \mathrm{~m}$.

8. Dept.: CHO, VAL

9. Nativa

10. Nombres vulgares: Mangosté, manguté(Valle del Cauca).

11. Nombres indígenas: No conocidos.

12. Usos: Depurativo (Valle del Cauca).

13. Nota: Sánchezet al. (2001:418) citaron en suanexo número 4 a Schwartzia colombiana (=Pseudosarcopera diazpiedrahitae) para el medio Caquetá(Amazonia colombiana). No obstante, esta especie es exclusiva del Chocó bogeográfico de Colombia y Ecuador(Giraldo-Cañas 2011).

\section{RESULTADOSYDISCUSIÓN}

Se registraron los ocho géneros conocidos para la familia (Giraldo-Cañas 2007), así como 55 especies, de las cuales once son endémicas (Tabla 1, Anexo 1). El género más rico fue Marcgravia L. con 19 especies (dos endémicas), seguido por Souroubea Aubl. (diez especies, una endémica), Marcgraviastrum (Wittm. ex Szyszyl.) de Roon y S. Dressler (nueve especies, dos endémicas), Schwartzia Vell. (siete especies, cinco endémicas), Sarcopera Bedell (cinco especies, ninguna endémica), Ruyschia Jacq. (tres especies, una endémica), Norantea Aubl. (una especie de amplia distribución neotropical) y Pseudosarcopera Gir.-Cañas (una especie del Chocó biogeográfico de Colombia y Ecuador). Así, Colombia se convierte en el país más rico en especies de esta familia, seguido por Brasil y Venezuela, con 39 especies (once endémicas) y 26 especies (ninguna endémica), respectivamente.

Las áreas naturales más diversas en Colombia son la región andina (41 especies), el Chocó biogeográfico (21), la Amazonia (16) y la Guayana (11)(Tabla 2). Le siguen como las regiones naturales menos diversas la Orinoquia (cinco especies), la región Caribe (4), el valle del río Magdalena (4), la Sierra Nevada de Santa Marta (3) y el valle del río Cauca (3). Estos datos de diversidad actual concuerdan con los análisis previamente considerados para las Marcgraviaceae (véase Giraldo-Cañas 1999). De las once especies endémicas de Colombia, la región andina presenta el mayor número, con ocho especies, una de las cuales (Souroubea intermedia de Roon) es compartida con el Chocó biogeográfico.

En cuanto a los gradientes altitudinales se refiere, la diversidad más alta se concentra en las tierras bajas $(0-500 \mathrm{~m})$ con 34 especies, seguido por el tercer intervalo altitudinal (1000-1500 m) con 31 especies, el segundo $(500-1000 \mathrm{~m})$ con 27 especies y por el cuarto (1500-2000 m) con 24 especies 
(Tabla 3). Cabe destacar que el límite altitudinal de la familia en Colombia corresponde a cerca de $2900 \mathrm{~m}$ para la especie Marcgravia brownei (Triana y Planch, Krug y Urb). En los bosques andinos por encima de los $2000 \mathrm{~m}$ de altitud, la diversidad de las Marcgraviaceae disminuye abruptamente, con ocho especies entre los 2000 my los 2500 m, y con tan sólo cuatro especies entre los $2500 \mathrm{~m}$ y los $3000 \mathrm{~m}$.

Por último, merece destacarse que con el desarrollo de esta investigación, se descubrieron diez nuevas especies y un nuevo género (Pseudosarcopera) para la ciencia (Picca y Giraldo-Cañas 1999, Giraldo-Cañas 2002a, 2003, 2007, 2011). Adicionalmente, se validaron cinco nuevas especies del género Marcgraviastrum (Roon y Giraldo-Cañas 2006, Giraldo-Cañas y Dressler 2011).

\section{CONCLUSIONES}

Se registraron ocho géneros y 55 especies, de las cuales once son endémicas. Por tanto, Colombia se convierte en el país más rico en especies de Marcgraviaceae, seguido por Brasil (39 especies, once endémicas) y Venezuela (26 especies, ninguna de las cuales es endémica). Las áreas naturales más diversas en Colombia son la región andina (41 especies), el Chocó biogeográfico (21), la Amazonia (16) y la Guayana (11). En cuanto a endemismo se refiere, la región andina presenta el mayor número de especies endémicas. Las áreas de tierras bajas húmedas a pluviales son las más ricas en especies. El límite altitudinal de la familia en Colombia corresponde a cerca de $2900 \mathrm{~m}$ para la especie Marcgravia brownei (Triana y Planch, Krug y Urb).

\section{AGRADECIMIENTOS}

El autor manifiesta su profundo reconocimiento al Instituto de Ciencias Naturales de la Universidad Nacional de Colombia por todas las facilidades que le brindó para la preparación de este trabajo. A los curadores de los herbarios CAUP, CEPEC, COAH, COL, CR, CUVC, CHOCO, F, HFAB, HUA, HUQ, IBGE,JAUM, MEDEL, MEXU,MO, MOL, MPU, NY, PSO, RSA, SI, SP, UDBC, UIS, US, VALLEy VEN por los préstamos enviados o por su grata colaboración durante la visita a sus instalaciones. A los herbarios de los jardines botánicos Rancho Santa Ana «RSA» (Claremont, California, EE.UU.) y Missouri «MO»(St. Louis, Missouri, EE.UU.), así como al Herbario Nacional de los EE.UU. (US, Smithsonian Institution, Washington D. C.) por las facilidades económicas brindadas para las visitas a sus instalaciones. A los doctores Paul M. Peterson (US), Orlando Rangel (COL) y Robert Soreng (US). Al Dr. Stefan Dressler (FR), por su valiosa y permanente colaboración y además, por la determinación, confirmación o corrección taxonómica de ejemplares durante su visita al Herbario Nacional Colombiano (COL) en el año
Tabla 3

\section{Diversidad de Marcgraviaceae por gradiente altitudinal en Colombia}

\begin{tabular}{lc}
\hline Gradiente altitudinal $(\mathbf{m})$ & Número de especies* \\
\hline $0-500$ & 34 \\
$500-1000$ & 27 \\
$1000-1500$ & 31 \\
$1500-2000$ & 24 \\
$2000-2500$ & 8 \\
$2500-3000$ & 4 \\
\hline Total & 55 \\
\hline
\end{tabular}

* La suma de la columna derecha es superior a 55 especies, porque muchas especies están presentes en varios gradientes altitudinales.

2009. Esta contribución se deriva del proyecto «Estudios sistemáticos en el complejo Norantea Aubl.(Marcgraviaceae). Parte II», de la Universidad Nacional de Colombia, sede Bogotá.

\section{LITERATURA CITADA}

APG III (The Angiosperm Phylogeny Group). 2009. An update of the Angiosperm Phylogeny Group classification for the orders and families of flowering plants: APG III. Bot J Linnean Soc. 161: 105-21.

Bedell, H. 1985. A generic revision of Marcgraviaceae I. The Norantea complex. PhD Dissertation. Maryland: College Park, University of Maryland.

Crisci, J. 1994. La especie: realidad y conceptos. p. 53-64. En: J. Llorente Bousquets, I. Luna (compiladores). Taxonomía biológica. México, DF: Universidad Autónoma de México, Fondo de Cultura Económica.

Cronquist, A. 1981. An integrated system of classification of flowering plants. Nueva York: Columbia Univ. Press. 1262 PP.

Cronquist, A. 1988. The evolution and classification of flowering plants. $2^{\mathrm{a}}$ ed. New York: The New York Botanical Garden, Bronx. 555 PP.

Delpino, F. 1869. Rivista monografica della famiglia delle Marcgraviaceae. Nuovo Giorn. Bot Ital. 1: 257-90.

de Roon, A. C. 1970. Marcgraviaceae. En: R. Woodson, R. Schery (Eds.). Fl. Panama. Ann Missouri Bot Gard. 57: 29-50.

de Roon, A. C. 1975. Contributions towards a monograph of the Marcgraviaceae. Tesis Doctoral, Universidad de Utrecht, Utrecht.

de Roon, A. C. 2005. A new species of Ruyschia (Marcgraviaceae) from the South American Andes. Novon. 15: 414-7.

de Roon, A. C., S. Dressler. 1997. New taxa of Norantea Aubl. s.1. (Marcgraviaceae) from Central America and adjacent South America. Bot Jahrb Syst. 119: 327-35.

de Roon, A. C., S. Dressler. 1999. New taxa of Sarcopera and Marcgraviastrum (Marcgraviaceae) from the Guayana Shield. Novon. 9: 248-52.

de Roon, A. C., D. Giraldo-Cañas. 2006. Contributions toward a monograph of Norantea complex (Marcgraviaceae, Ericales): Validation of four new species for Marcgraviastrum. Caldasia. 28 (1): 15-22.

Dressler, S. 2000. A new species of Marcgravia (Marcgraviaceae) from Amazonia with some notes on the Galeatae group including a key. Willdenowia. 30 (2): 369-74.

Dressler, S. 2001. Marcgraviaceae. En: J. Steyermark, P. Berry, B. Holst (Eds). Fl. Venezuelan. Guayana. 6: 248-60. 


\section{Bioetnia Volumen 8 No 1 (enero-junio), 2011}

Etter, A., W. van Wyngaarden. 2000. Patterns of landscape transformation in Colombia, with emphasis in the Andean Region. Ambio. 29 (7): 432-9.

Ewan, J. 1951. Taxonomic notes on various species of Musaceae, Marcgraviaceae, Guttiferae and Solanaceae of Colombia. Nat Hist Misc. 88: 1-9.

Ferreira, G. 1995. Estudo taxonômico das espécies brasileiras do gênero Norantea Aublet (Marcgraviaceae). Arq Jard Bot. 33: 9-53.

Font Quer, P. 1964. Botánica pintoresca. Barcelona: Ed. Ramón Sopena SA. 719 pp.

Gilg, E., E. Werdermann. 1925. Marcgraviaceae. En: Engler, A. K. Prantl(Eds). Nat Pflanzenfam. 21: 94-106.

Giraldo-Cañas, D. 1999. Las Marcgraviaceae de la Amazonia colombiana: estudio preliminar sobre su diversidad, distribución y fitogeografía. Darwiniana. 37 (1-2): 15-24.

Giraldo-Cañas, D. 2002a. Novedades taxonómicas y corológicas en Marcgraviastrum, Sarcopera y Schwartzia (Marcgraviaceae) de Sudamérica. Hickenia. 32: 119-23.

Giraldo-Cañas, D. 2002b. Una nueva especie de Marcgravia, grupo Galeatae (Marcgraviaceae) de la cordillera Oriental y catálogo del género para Colombia. Rev Acad Colomb Cien Exact. 26 (100): 367-71.

Giraldo-Cañas, D. 2003. Revisión de las especies colombianas del género Schwartzia (Marcgraviaceae). Caldasia. 25 (1): 1-21.

Giraldo-Cañas, D. 2005. Validation of a new species of Schwartzia (Marcgraviaceae) and synopsis of the genus for Ecuador. Novon. 15: 123-7.

Giraldo-Cañas, D. 2007. Un nuevo género de la familia neotropical Marcgraviaceae (Ericales) y circunscripción del Complejo Norantea. Caldasia. 29 (2): 203-17.

Giraldo-Cañas, D. 2010. Distribución e invasión de gramíneas $\mathrm{C}_{3} \mathrm{y} \mathrm{C}_{4}$ (Poaceae) en un gradiente altitudinal de los Andes de Colombia. Caldasia. 32 (1): 65-86.

Giraldo-Cañas, D. 2011. Estudios morfológicos y taxonómicos en el complejo Norantea (Marcgraviaceae): Revisión de Norantea, Pseudosarcopera y Schwartzia. Bogotá: Instituto de Ciencias Naturales, Universidad Nacional de Colombia. p. 1-172.

Giraldo-Cañas, D., S. Dressler. 2011. Validation of a new species of Marcgraviastrum from Colombia (Norantea Complex, Marcgraviaceae). Caldasia. 33: 21-7.

Giraldo-Cañas, D., P. Fiaschi. 2005. Las Marcgraviaceae (Ericales) de Brasil: Las especies del complejo Norantea. Caldasia. 27 (2): 173-94.

Giraldo-Cañas, D., P. Picca. 1999. Tres nuevos registros de Marcgravia (Marcgraviaceae) para la flora mexicana. Hickenia. 2 (62): 289-92.

Grytnes, J. A., O. R. Vetaas. 2002. Species richness and altitude: a comparison between Null Models and interpolated plant species richness along the Himalayan altitudinal gradient, Nepal. Am Nat. 159 (3): 294-304.

Hallier, H. 1905. Provisional scheme of the natural (phylogenetic) system of Flowering Plants. New Phytol. 4 (7): 151-62.

Hammel, B. E. 2006. Three new species of Marcgraviaceae from Costa Rica, with references to related species and notes on the generic placement of Schwartzia jimenezii. Lankesteriana. 6 (2): 73-81.

Holmgren, P., N. Holmgren, L. Barnett. 1990. Index Herbariorum. Part I: The Herbaria of the World. Nueva York: The New York Botanical Garden. 693 pp.

Johnston, I. M. 1941. Preparación de ejemplares botánicos para herbario. Tucumán: Instituto Miguel Lillo, Universidad Nacional de Tucumán. 59 pp.

Jørgensen, P. M., C. Ulloa Ulloa, B. León, S. León-Yánez, S. G. Beck, M. Nee, et al. 2011. Regional patterns of vascular plant diversity and endemism. p. 192-203. En: S. K. Herzog, R. Martínez, P. M. Jørgensen, H. Tiessen
(Eds). Climate change and biodiversity in the Tropical Andes. São José dos Campos: Inter-American Institute for Global Change Research (IAI) and Scientific Committee on Problems of the Environment (SCOPE).

Kessler, M. 2002. The elevational gradient of Andean plant endemism: varying influences of taxon-specific traits and topography at different taxonomic levels. J Biogeograp. 29: 1159-65.

Kuntze, O. 1891. Ternstroemiaceae. Revis Gen Pl. 1: 61-5.

Lægaard, S. 1999. Biogeography of Ecuadorian grasses. Arnaldoa. 6 (2): 18596.

Larsen, T. H., G. Brehm, H. Navarrete, P. Franco, H. Gómez, J. L. Mena, et al. 2011a. Range shifts and extintions driven by climate change in the Tropical Andes: Synthesis and directions. p. 47-67. En: S. K. Herzog, R. Martínez, P. M. Jørgensen, H. Tiessen (Eds). Climate change and biodiversity in the Tropical Andes. São José dos Campos: Inter-American Institute for Global Change Research (IAI) and Scientific Committee on Problems of the Environment (SCOPE).

Larsen, T. H., F. Escobar, I. Armbrecht. 2011b. Insects of the Tropical Andes: Diversity patterns, processes and global change. p. 228-44. En: S. K. Herzog, R. Martínez, P. M. Jørgensen, H. Tiessen (Eds). Climate change and biodiversity in the Tropical Andes. São José dos Campos: InterAmerican Institute for Global Change Research (IAI) and Scientific Committee on Problems of the Environment (SCOPE).

Lawrence, G. H. M. 1962. An introduction to plant taxonomy. Nueva York: The Macmillan Co. 428 pp.

McDade, L. A. 1995. Species concepts and problems in practice: insight from botanical monographs. Syst Bot. 20 (4): 606-22.

Oommen, M. A., K. Shanker. 2005. Elevational species richness patterns emerge from multiple local mechanisms in Himalayan woody plants. Ecology. 86 (11): 3039-47

Picca P., D. Giraldo-Cañas. 1999. Una nueva especie de Marcgravia (Marcgraviaceae) de la Amazonia colombiana. Hickenia. 63: 293-7.

Sánchez, M., Á. Duque, P. Miraña, E. Miraña, J. Miraña. 2001. Valoración del uso no comercial del bosque-métodos en etnobotánica cuantitativa. p. 179224, p. 399-430. En: J. F. Duivenvoorden, H. Balslev, J. Cavelier, C. Grandez, H. Tuomisto, R. Valencia (Eds.). Evaluación de recursos vegetales no maderables en la Amazonia noroccidental. Amsterdam: Institute for Biodiversity and Ecosystem Dynamics IBED, Universiteit van Amsterdam.

Schultes, R. 1951. Contribución al conocimiento de la flora amazónica de Colombia, I. Rev Acad Colomb Cien Exact. 8 (31): 397-408.

Stevens, G. C. 1992. The elevational gradient in altitudinal range: an extension of Rapoport's latitudinal rule to altitude. Am Nat. 140 (6): 893-911.

Suárez Mayorga, Á., J. D. Lynch. 2008. Anfibios de la región del transecto Sumapaz. En: T. van der Hammen (Ed). Estudios de Ecosistemas Tropandinos: La cordillera Oriental colombiana-Transecto Sumapaz. Sttugart: J. Cramer. p. 311-26.

Triana, J., J. Planchon. 1862. Prodromus Floræ Novo-Granatensis: Ternstroemiaceae trib. I. Marcgravieae. Ann Sci Nat Bot., sér. 4, 17: 35979.

Uribe Meléndez, J. 2008. Monografía de Frullania subgénero Meteoriopsis (Frullaniaceae, Marchantiophyta). Caldasia. 30 (1): 49-94.

Ward, N., R. Price. 2002. Phylogenetic relationships of Marcgraviaceae: Insights from three chloroplast genes. Syst Bot. 27: 149-60.

Wiens, J. J., M. R. Servedio. 2000. Species delimitation in systematics: inferring diagnostic differences between species. Proc R Soc. (London) $B$ 267: 631-6.

Wittmack, L. 1878. Marcgraviaceae. Fl Brasil. 12: 213-58. 


\section{Anexo 1}

\section{Catálogo de las Marcgraviaceae de Colombia (Ocho géneros, 55 especies, 11 especies endémicas)}

Caracasia Szyszyl.

= Ruyschia Jacq.

Caracasia tremadena (Ernst) Szyszyl.

= Ruyschia tremadena (Ernst) Lundell

Marcgravia L.

19 especies (2 endémicas).

Marcgravia affinis Hemsl.

= Marcgravia caudata Triana \& Planch.

Marcgravia apionifera de Roon (in sched.)

= Marcgravia roonii S. Dressler

\section{Marcgravia atropunctata de Roon}

Ref.: E. Forero 2695 (COL).

Andes, Chocó Biogeográfico

0-1500 m.

Dept.: ANT, CHO, NAR, VAL

Nativa

Marcgravia brownei (Triana \& Planch.) Krug \& Urb.

Sin.: Marcgravia rectiflora Triana \& Planch. var. brownei Triana \& Planch.

Ref.: D. Giraldo-Cañas 5027 (COL).

Andes, Sierra Nevada de Santa Marta

1000-3000 m.

Dept.: ANT, BOY, CAL, CAU, CES, CHO, CUN, HUI, MAG, NAR, NSA, PUT, QUI, RIS, VAL

Nativa

Marcgravia caudata Triana \& Planch.

Sin.: Marcgravia affinis Hemsl.

Ref.: D. Giraldo-Cañas 1979-a (COL).

Andes, Chocó Biogeográfico

0-2000 m.

Dept.: ANT, CAU, CHO, NAR

Nativa

\section{Marcgravia coriacea Vahl}

Registro basado en determinaciones erróneas, las cuales, en su mayoría, corresponden a Marcgravia crenata Poepp. ex Wittm.

Marcgravia crenata Poepp. ex Wittm.

Sin.: Marcgravia macrophylla (Wittm.) Gilg, Marcgravia rectiflora Triana \& Planch. var. macrophylla Wittm.

Ref.: H. Mendoza 67 (CUVC).

Amazonia, Andes, Guayana, Llanura del Caribe, Orinoquia,

Chocó Biogeográfico, valle del Magdalena

0-1000 m.

Dept.: AMA, ANT, CAQ, CHO, MET, PUT, SAN

Nativa

Marcgravia dressleri Gir.-Cañas

Ref.: R. Galindo 325 (COL).

Andes

1000-2000 m.

Dept.: ANT, SAN

Endémica
Marcgravia eichleriana Wittm.

Ref.: R. E. Schultes 10105-C (COL).

Amazonia, Andes

0-2000 m.

Dept:: AMA, ANT, CAU, VAU

Nativa

Marcgravia fosbergiana J. Ewan

Ref.: J. A. Ewan 16053 (COL, US, NO), estos ejemplares no los he podido localizar; fuente Ewan (1951).

Andes

1000-1500 m.

Dept.: NAR

Nota: Estatus sin definir (esta especie no fue incluida en los análisis de riqueza).

Marcgravia glandulosomarginata Hammel

Ref.: Á. Cogollo 2903 (COL, JAUM, MO).

Andes

500-2000 m.

Dept.: ANT

Nativa

Marcgravia goudotiana (Triana \& Planch.) de Roon

Sin.: Marcgravia rectiflora Triana \& Planch. var. goudotiana Triana \& Planch.

Nota: Estatus sin definir (esta especie no fue incluida en los análisis de riqueza).

Marcgravia longifolia J. F. Macbr.

Ref.: R. E. Schultes 6967 (COL).

Amazonia, Guayana

0-500 m.

Dept.: AMA, CAQ

Nativa

Usos: Los indígenas Yukuna (Amazonia) emplean la savia como analgésico (observaciones personales).

Marcgravia macrophylla (Wittm.) Gilg

= Marcgravia crenata Poepp. ex Wittm.

Marcgravia membranacea Standl.

= Marcgravia nervosa Triana \& Planch.

Marcgravia myriostigma Triana \& Planch.

Ref.: E. P. Killip 39100 (COL).

Llanura del Caribe, Chocó Biogeográfico

0-2000 m.

Dept.: ANT, CHO

Nativa

Marcgravia nepenthoides Seem.

Ref.: J. Brand 32 (COL, MO).

Andes, Llanura del Caribe

0-1000 m.

Dept.: ANT, RIS

Nativa

Marcgravia nervosa Triana \& Planch.

Sin.: Marcgravia membranacea Standl.

Ref.: D. Giraldo-Cañas 1918-a (COL). 


\section{Bioetnia Volumen 8 No 1 (enero-junio), 2011}

\section{Anexo 1 \\ Catálogo de las Marcgraviaceae de Colombia (Ocho géneros, 55 especies, 11 especies endémicas) (continuación)}

\author{
Andes \\ 500-1500 m \\ Dept.: ANT, CHO, NAR \\ Nativa \\ Marcgravia parviflora Rich. ex Wittm. \\ = Marcgravia pedunculosa Triana \& Planch. \\ Marcgravia parviflora Rich. ex Wittm. var. sprucei Wittm. \\ = Marcgravia sprucei (Wittm.) Gilg
}

Marcgravia pedunculosa Triana \& Planch.

Sin.: Marcgravia parviflora Rich. ex Wittm.

Ref.: D. Giraldo-Cañas 2084-a (COL).

Amazonia, Andes, Orinoquia

$0-1500 \mathrm{~m}$.

Dept.: AMA, ANT, CAQ, MET, PUT

Nativa

Marcgravia pentandra Ruiz \& Pav. ex E. A. López

= Ruyschia pavonii G. Don

Marcgravia rectiflora Triana \& Planch. var. brownei Triana \& Planch.

$=$ Marcgravia brownei (Triana \& Planch.) Krug \& Urb.

Marcgravia rectiflora Triana \& Planch. var. goudotiana Triana \& Planch.

= Marcgravia goudotiana (Triana \& Planch.) de Roon

Marcgravia rectiflora Triana \& Planch. var. macrophylla Wittm.

= Marcgravia crenata Poepp. ex Wittm.

Marcgravia roonii S. Dressler

Sin.: Marcgravia apionifera de Roon (in sched.)

Ref.: E. P. Killip 34790 (COL).

Andes, Chocó Biogeográfico

0-2000 m.

Dept.: ANT, BOY, CHO, CUN, NSA, RIS, SAN, VAL

Nativa

Marcgravia sprucei (Wittm.) Gilg

Sin.: Marcgravia parviflora Rich. ex Wittm. var. sprucei Wittm.

Ref.: J. Cuatrecasas 7227 (COL).

Amazonia, Andes

$0-1000 \mathrm{~m}$.

Dept.: AMA, ANT, PUT, VAU

Nativa

Marcgravia weberbaueri Gilg

Ref.: D. D. Soejarto 3504 (COL).

Andes

500-2000 m

Dept.: ANT, CHO, NAR, PUT

Nativa

Marcgravia williamsii J. F. Macbr. Ref.: E. P. Killip 39100 (COL).

Amazonia, Andes, Chocó Biogeográfico $0-1500 \mathrm{~m}$.
Dept: CHO, PUT, VAL

Nativa

Usos: Analgésico (comunidad Waunana, Chocó).

Marcgravia yukunarum Picca \& Gir.-Cañas

Ref.: E. Álvarez 1104 (COAH, COL, JAUM).

Amazonia

0-500 m.

Dept.: AMA

Endémica

Marcgravia zonopunctata S. Dressler

Ref.: G. A. Black 46-339 (IAN), ejemplar no visto, fuente

Dressler (2000)

Amazonia

0-500 m.

Dept.: AMA

Nativa

Marcgraviastrum (Wittm. ex Szyszyl.) de Roon \& S.

Dressler

9 especies (2 endémicas).

Marcgraviastrum apaporensis de Roon \& Bedell

Ref.: R. E. Schultes 14589 (COL, US).

Amazonia, Guayana

0-500 m.

Dept.: AMA, VAU

Endémica

Marcgraviastrum elegans de Roon \& Bedell ex Gir.-

Cañas \& S. Dressler

Ref.: J. L. Zarucchi 2210 (COL, US).

Guayana

0-500 m.

Dept.: GUA, VAU

Endémica

Marcgraviastrum gigantophyllum (Gilg) Bedell ex S.

Dressler

Sin.: Norantea gigantophylla Gilg

Ref.: J. Luteyn 10672 (NY).

Chocó Biogeográfico

0-500 m

Dept.: $\mathrm{CHO}$

Nativa

Marcgraviastrum macrocarpum (G. Don) Bedell ex S.

Dressler

Sin.: Norantea eoetvoesorum Al. Richt., Norantea eoetvoesorum V. A. Richt., Norantea macrocarpa G. Don, Norantea pardoana Weberb. \& Gilg

Ref.: D. Giraldo-Cañas 5026 (COL).

Andes

1500-3000 m

Dept.: CUN, BOY, SAN

Nativa

Nombres vulgares: Bailador (Santander), trompo

(Cundinamarca) 


\section{Anexo 1 \\ Catálogo de las Marcgraviaceae de Colombia (Ocho géneros, 55 especies, 11 especies endémicas) (continuación)}

Marcgraviastrum mixtum (Triana \& Planch.) Bedell

Sin.: Norantea curva J. F. Macbr., Norantea mixta Triana \& Planch., Norantea peduncularis Poepp. ex Wittm., Norantea uleana Pilg.

Ref.: D. Giraldo-Cañas 3306 (COL).

Andes, Guayana, Orinoquia, Chocó Biogeográfico, valle del Cauca, valle del Magdalena

0-2000 m.

Dept.: ANT, BOY, CAQ, CAU, CHO, CUN, GUA, HUI, MET, NAR, PUT, QUI, SAN, TOL, VAL, VAU

Nativa

Usos: Cestería (Santander).

\author{
Marcgraviastrum pauciflorum de Roon \& Bedell \\ Ref.: A. Gentry 40737 (COL, MO). \\ Andes, Chocó Biogeográfico \\ 0-1000 m. \\ Dept.: $\mathrm{CHO}, \mathrm{VAL}$ \\ Nativa
}

Marcgraviastrum sodiroi (Gilg) Bedell ex S. Dressler

Sin.: Norantea sodiroi Gilg

Ref.: D. Giraldo-Cañas 1937-a (COL).

Andes

500-2000 m

Dept.: NAR

Nativa

Marcgraviastrum subsessile (Benth.) Bedell

Sin.: Norantea subsessilis (Benth.) Donn. Sm., Ruyschia subsessilis Benth.

Ref.: E. P. Killip 33217 (COL, US)).

Andes, Chocó Biogeográfico

0-1000 m.

Dept.: ANT, CAU, CHO

Nativa

Marcgraviastrum vogelii de Roon \& Bedell

Ref.: L. E. Mora 4148 (COL).

Andes, Chocó Biogeográfico

500-2000 m.

Dept.: CHO, NAR, VAL

Nativa

Norantea Aubl.

1 especie

Norantea anomala Kunth

= Sarcopera anomala (Kunth) Bedell

Norantea aurantiaca Spruce ex Gilg

= Sarcopera aurantiaca (Spruce ex Gilg) de Roon \& S.

Dressler

Norantea caccabion Delpino

= Sarcopera cordachida (Ruiz \& Pav. ex G. Don) Bedell ex

S. Dressler

Norantea cordachida Ruiz \& Pav. ex G. Don
= Sarcopera cordachida (Ruiz \& Pav. ex G. Don) Bedell ex S. Dressler

Norantea curva J. F. Macbr.

= Marcgraviastrum mixtum (Triana \& Planch.) Bedell

Norantea droseriformis Rusby ex Gilg \& Werderm.

= Sarcopera anomala (Kunth) Bedell

Norantea eoetvoesorum Al. Richt.

= Marcgraviastrum macrocarpum (G. Don) Bedell ex $\mathbf{S}$.

Dressler

Norantea eoetvoesorum V. A. Richt.

= Marcgraviastrum macrocarpum (G. Don) Bedell ex S.

Dressler

Norantea gigantophylla Gilg

= Marcgraviastrum gigantophyllum (Gilg) Bedell ex S.

Dressler

Norantea gracilis (Wittm.) V. A. Richt.

= Norantea guianensis Aubl.

Norantea guianensis Aubl.

Sin.: Norantea gracilis (Wittm.) V. A. Richt., Norantea japurensis Mart., Norantea zelodoniana Standl. \& L. O. Williams Ref.: D. Giraldo-Cañas 2164 (COL, MO).

Amazonia, Andes, Guayana, Orinoquia, Chocó Biogeográfico, valle del Cauca, valle del Magdalena

0-2000 m.

Dept.: AMA, ANT, CAQ, CHO, GUI, MET, PUT, QUI, SAN, VAL,

VAU, VIC

Nativa

Nombres vulgares: Bejuco rabo de guacamaya, trompillo.

Nombres indígenas: Ma-haí-ko-ì, mahaí-komì (lengua Taiwano,

Amazonas, Vaupés), ñá-eoo-ka-wa-pee, ñárookawapee (lengua Karapana, Amazonas, Vaupés), koo-eé-aw-ì-ro (lengua Kurripako, Amazonas, Vaupés), hó-po-choo (lengua Puinave, Amazonas, Vaupés).

Usos: Cestería (Santander); por otra parte, su savia se emplea para aliviar quemaduras y lesiones cutáneas. En algunas fincas se le conserva como ornamental por el destacado color rojo y el tamaño de sus inflorescencias.

Norantea japurensis Mart.

= Norantea guianensis Aubl.

Norantea macrocarpa G. Don

= Marcgraviastrum macrocarpum (G. Don) Bedell ex S.

Dressler

Norantea microscypha Gilg

= Sarcopera anomala (Kunth) Bedell

Norantea mixta Triana \& Planch.

= Marcgraviastrum mixtum (Triana \& Planch.) Bedell

Norantea pardoana Weberb. \& Gilg 


\section{Bioetnia Volumen 8 № 1 (enero-junio), 2011}

\section{Anexo 1 \\ Catálogo de las Marcgraviaceae de Colombia (Ocho géneros, 55 especies, 11 especies endémicas) (continuación)}

\author{
= Marcgraviastrum macrocarpum (G. Don) Bedell ex S. \\ Dressler \\ Norantea peduncularis Poepp. ex Wittm. \\ = Marcgraviastrum mixtum (Triana \& Planch.) Bedell \\ Norantea rosulata (de Roon \& Bedell) Hammell (inédito) \\ = Sarcopera rosulata de Roon \& Bedell \\ Norantea sandiensis Gilg \\ = Schwartzia weddelliana (Baill.) Bedell
}

Nota: Especie citada para Colombia por Schultes (1951: 404, sub Norantea sandiensis Gilg); registro basado en material mal determinado.

Norantea sessiliflora Triana \& Planch.

= Sarcopera sessiliflora (Triana \& Planch.) Bedell

Norantea sessilis L. O. Williams

= Sarcopera sessiliflora (Triana \& Planch.) Bedell

Norantea sodiroi Gilg

= Marcgraviastrum sodiroi (Gilg) Bedell ex S. Dressler

Norantea subsessilis (Benth.) Donn. Sm.

= Marcgraviastrum subsessile (Benth.) Bedell

Norantea uleana Pilg.

= Marcgraviastrum mixtum (Triana \& Planch.) Bedell

Norantea zahlbruckneri V. A. Richt.

= Sarcopera anomala (Kunth) Bedell

Norantea zelodoniana Standl. \& L. O. Williams

$=$ Norantea guianensis Aubl.

Pseudosarcopera Gir.-Cañas

1 especie

Pseudosarcopera diaz-piedrahitae (Gir.-Cañas) Gir.Cañas

Sin.: Schwartzia colombiana de Roon \& Bedell, Schwartzia diaz-piedrahitae Gir.-Cañas

Ref.: M. Monsalve 484 (COL, JAUM, MO, NY).

Chocó Biogeográfico

0-500 m.

Dept.: CHO, VAL

Nativa

Nombres vulgares: Mangosté, manguté (Valle del Cauca).

Usos: Depurativo (Valle del Cauca).

Nota: Sánchez et al. (2001: 418) citaron en su anexo número 4 a Schwartzia colombiana (= Pseudosarcopera diaz-

piedrahitae) para el medio Caquetá (Amazonia colombiana). No obstante, esta especie es exclusiva del Chocó Biogeográfico de Colombia y Ecuador (Giraldo-Cañas 2011).

Ruyschia Jacq.

3 especies (1 endémica)
Sin.: Caracasia Szyszyl., Vargasia Ernst

Ruyschia amazonica Mart. var. dilatata Triana \& Planch.

= Souroubea sympetala Gilg

Ruyschia bicolor Benth.

= Souroubea bicolor (Benth.) de Roon

Ruyschia corallina Mart.

= Souroubea corallina (Mart.) de Roon

Ruyschia crassipes Triana \& Planch.

= Souroubea crassipes (Triana \& Planch.) Wittm.

Ruyschia guianensis (Aubl.) Vitman

= Souroubea guianensis Aubl.

Ruyschia pavonii G. Don

Sin.: Marcgravia pentandra Ruiz \& Pav. ex E. A. López,

Ruyschia sphaeradenia Delpino

Ref.: D. Sánchez 567 (COL).

Andes

1500-2000 m.

Dept.: ANT, CHO, RIS

Nativa

Ruyschia pilophora Triana \& Planch.

Ref.: D. Giraldo-Cañas 4964 (COL).

Andes

1500-3000 m

Dept.: ANT, CAL, VAL

Endémica

Ruyschia souroubea Sw.

= Souroubea guianensis Aubl.

Ruyschia sphaeradenia Delpino

= Ruyschia pavonii G. Don

Ruyschia tremadena (Ernst) Lundell

Sin.: Caracasia tremadena (Ernst) Szyszyl., Ruyschia

zuluetana Philipson, Vargasia tremadena Ernst

Ref.: D. Giraldo-Cañas 3574 (COL).

Andes, Guayana, Chocó Biogeográfico, Sierra Nevada de Santa Marta

0-2500 m

Dept.: CUN, MAG, MET, VAL

Nativa

Ruyschia subsessilis Benth.

= Marcgraviastrum subsessile (Benth.) Bedell

Ruyschia zuluetana Philipson

= Ruyschia tremadena (Ernst) Lundell

Sarcopera Bedell

5 especies 


\section{Anexo 1 \\ Catálogo de las Marcgraviaceae de Colombia (Ocho géneros, 55 especies, 11 especies endémicas) (continuación)}

\author{
Sarcopera anomala (Kunth) Bedell \\ Sin.: Norantea anomala Kunth, Norantea droseriformis Rusby \\ ex Gilg \& Werderm., Norantea microscypha Gilg, Norantea \\ zahlbruckneri V. A. Richt. \\ Ref.: D. Giraldo-Cañas 2131-a (COL). \\ Andes \\ 1000-2500 m. \\ Dept.: ANT, CAU, NAR, PUT, SAN, VAL \\ Nativa \\ Sarcopera aurantiaca (Spruce ex Gilg) de Roon \& S. \\ Dressler \\ Sin.: Norantea aurantiaca Spruce ex Gilg \\ Ref.: D. Giraldo-Cañas 4576 (COL). \\ Amazonia, Guayana \\ 0-500 m. \\ Dept.: AMA, CAQ, VAU \\ Nativa \\ Nombre indígena: Ma-pae-kó-há-rì-nee-ka (lengua Kararaná, \\ Amazonas, Vaupés).
}

Sarcopera cordachida (Ruiz \& Pav. ex G. Don) Bedell ex S. Dressler

Sin.: Norantea caccabion Delpino, Norantea cordachida Ruiz \& Pav. ex G. Don

Ref.: D. Giraldo-Cañas 4631 (COL).

Andes, Chocó Biogeográfico

0-1500 m.

Dept.: ANT, CHO, NAR, RIS, VAL

Nativa

\author{
Sarcopera rosulata de Roon \& Bedell \\ Sin.: Norantea rosulata (de Roon \& Bedell) Hammell (inédito) \\ Ref.: R. Callejas 8732 (HUA, NY) \\ Andes \\ 1000-1500 m. \\ Dept.: ANT \\ Nativa
}

Sarcopera sessiliflora (Triana \& Planch.) Bedell

Sin.: Norantea sessiliflora Triana \& Planch., Norantea sessilis L. O. Williams

Ref.: J. Idrobo 1418 (COL, US).

Andes, Chocó Biogeográfico

$0-1500 \mathrm{~m}$.

Dept.: NAR, VAL

Nativa

Schwartzia Vell.

7 especies ( 5 endémicas).

\section{Schwartzia andina Gir.-Cañas}

Ref.: R. Callejas 11073 (COL, HUA, MO).

Andes

1500-2500 m.

Dept.: ANT, CAL, CAU

Endémica
Schwartzia antioquensis Gir.-Cañas

Sin.: Schwartzia sclerophylla de Roon \& Bedell, nom. nud.

Ref.: R. Fonnegra 5569 (COL, HUA, MO).

Andes

1000-2000 m.

Dept.: ANT

Endémica

Nombre vulgar: Oreja de toro.

\section{Schwartzia chocoensis Gir.-Cañas}

Sin.: Schwartzia foreroi de Roon \& Bedell, nom. nud.

Ref.: E. Forero 3000 (CHOCO, COL, MO).

Andes, Chocó Biogeográfico

0-1500 m.

Dept.: CHO, VAL

Nativa

Schwartzia colombiana de Roon \& Bedell

= Pseudosarcopera diaz-piedrahitae (Gir.-Cañas) Gir.-

Cañas

Schwartzia diaz-piedrahitae Gir.-Cañas

= Pseudosarcopera diaz-piedrahitae (Gir.-Cañas) Gir.-

Cañas

Schwartzia foreroi de Roon \& Bedell

= Schwartzia chocoensis Gir.-Cañas

Schwartzia lozaniana Gir.-Cañas

Sin.: Schwartzia venusta Bedell (in sched.), Schwartzia venusta de Roon \& Bedell (in sched.).

Ref.: J. Luteyn 6880 (COL, MO, NY).

Andes

500-2000 m.

Dept.: NAR

Nativa

Schwartzia micradenia de Roon \& Bedell

= Schwartzia renvoizei Gir.-Cañas

Schwartzia mutisii de Roon \& Bedell

Nota: Este binomio no ha sido publicado, pero aparece citado en la base de datos del herbario US -basado en el espécimen Mutis 4455 (recolectado en territorio colombiano)-, pero hasta el presente dicho ejemplar no ha sido localizado (Giraldo-Cañas 2011).

Schwartzia parrae Gir.-Cañas

Ref.: R. Callejas 7957 (HUA, MO, NY).

Andes

1000-2000 m.

Dept.: ANT

Endémica

Schwartzia petersonii Gir.-Cañas

Ref.: D. Canal Gallego 98 (COL).

Andes 


\section{Bioetnia Volumen 8 No 1 (enero-junio), 2011}

\section{Anexo 1 \\ Catálogo de las Marcgraviaceae de Colombia (Ocho géneros, 55 especies, 11 especies endémicas) (continuación)}

1000-2500 m.

Dept.: ANT, CHO

Endémica

Schwartzia pterosara de Roon \& Bedell ex Gir.-Cañas Nota: Bedell (1985) citó esta especie para Colombia (Cauca), pero hasta el momento no se ha documentado su presencia en el país (Giraldo-Cañas 2005, 2011).

\section{Schwartzia renvoizei Gir.-Cañas}

Sin.: Schwartzia micradenia de Roon \& Bedell (in sched.)

Ref.: J. Cuatrecasas 15456 (COL, F, US).

Andes

1000-1500 m.

Dept.: VAL

Endémica

Schwartzia sclerophylla de Roon \& Bedell

= Schwartzia antioquensis Gir.-Cañas

Schwartzia venusta Bedell

= Schwartzia lozaniana Gir.-Cañas

Schwartzia venusta de Roon \& Bedell

= Schwartzia lozaniana Gir.-Cañas

Schwartzia weddelliana (Baill.) Bedell

Nota: Especie citada para Colombia por Schultes (1951: 404, sub Norantea sandiensis Gilg); registro basado en material mal determinado.

Souroubea Aubl.

10 especies (1 endémica).

Souroubea auriculata Delpino

= Souroubea guianensis Aubl.

Souroubea bicolor (Benth.) de Roon

Sin.: Ruyschia bicolor Benth.

Ref.: D. Giraldo-Cañas 5002 (COL).

Amazonia, Andes, Chocó Biogeográfico

$0-2000 \mathrm{~m}$.

Dept.: ANT, CAU, CHO, NAR, PUT, TOL, VAL

Nativa

Souroubea corallina (Mart.) de Roon

Sin.: Ruyschia corallina Mart., Souroubea guianensis Aubl. var. corallina (Mart.) Wittm.

Ref.: D. Giraldo-Cañas 4561 (COL).

Amazonia, Guayana

$0-500 \mathrm{~m}$.

Dept.: AMA, CAQ, GUA, VAU

Nativa

Souroubea crassipes (Triana \& Planch.) Wittm.

Sin.: Ruyschia crassipes Triana \& Planch.

Ref.: J. Betancur 11054 (COL).

Andes
1500-2000 m

Dept.: CUN, SAN

Nativa

Souroubea crassipetala de Roon

Ref.: R. E. Schultes 16912 (COL, US).

Amazonia

0-500 m

Dept.: AMA, VAU

Nativa

Souroubea dasystachya Gilg \& Werderm.

Ref.: J. Duque-Jaramillo 2038 (COL).

Amazonia

$0-500 \mathrm{~m}$

Dept.: AMA

Nativa

\section{Souroubea fragilis de Roon}

Ref.: J. Zarucchi 6156 (HUA, MO, ejemplares determinados por $\mathrm{H}$. Bedell); ejemplares no vistos, fuente base de datos Tropicos del herbario $\mathrm{MO}$.

Andes

2000-3000 m

Dept.: ANT

Nativa

Souroubea guianensis Aubl.

Sin.: Ruyschia guianensis (Aubl.) Vitman, Ruyschia souroubea

Sw., Souroubea auriculata Delpino

Ref.: D. Giraldo-Cañas 4574 (COL).

Amazonia, Guayana

$0-500 \mathrm{~m}$.

Dept.: AMA, CAQ, GUA, GUI, VAU

Nativa

Nombre indígena: Itorao (lengua Uitoto, Amazonia).

Usos: Los indígenas Uitoto emplean la savia para curar la gripa y la ronquera.

Souroubea guianensis Aubl. var. corallina (Mart.) Wittm.

= Souroubea corallina (Mart.) de Roon

Souroubea guianensis Aubl. var. dilatata (Triana \& Planch.)

Wittm.

= Souroubea sympetala Gilg

Souroubea guianensis Aubl. var. spectabilis Wittm.

= Souroubea sympetala Gilg

Souroubea intermedia de Roon

Ref.: E. P. Killip 39146 (COL, F, MO, US).

Andes, Chocó Biogeográfico

0-1500 m

Dept.: CHO, NAR, VAL

Endémica 


\section{Anexo 1}

\section{Catálogo de las Marcgraviaceae de Colombia (Ocho géneros, 55 especies, 11 especies endémicas) (continuación)}

Souroubea sympetala Gilg

Sin.: Ruyschia amazonica Mart. var. dilatata Triana \& Planch., Souroubea guianensis Aubl. var. dilatata (Triana \& Planch.)

Wittm., Souroubea guianensis Aubl. var. spectabilis Wittm.

Ref.: D. Giraldo-Cañas 3307 (COL).

Andes, Guayana, Llanura del Caribe, Orinoquia, Chocó

Biogeográfico, Sierra Nevada de Santa Marta, valle del Cauca, valle del Magdalena

0-1500 m.

Dept.: ANT, BOY, CAL, CHO, CUN, GUA, GUJ, MAG, MET, NSA, SAN

Nativa
Souroubea vallicola Woodson ex de Roon

Ref.: Duke \& Idrobo $11275(\mathrm{U})$, ejemplar no visto, fuente de Roon (1975: 167).

Chocó Biogeográfico

$0-500 \mathrm{~m}$.

Dept.: $\mathrm{CHO}$

Nativa

Vargasia Ernst

= Ruyschia Jacq.

Vargasia tremadena Ernst

= Ruyschia tremadena (Ernst) Lundell 